\title{
Los roles del ambiente, de la higiene, de la epidemiología y de las vacunas en el desafío de las enfermedades infecciosas
}

\author{
Felipe Cabello C.
}

\section{The roles that the environment, the sanitary conditions, the epidemiology and vaccines play in the challenge of infectious diseases}

This commentary uses a recent article in the press about the failure of the Gates Foundation's plans to eradicate poliomyelitis in Asia and Africa as a basis for discussing the often limited historical role that vaccines have played in controlling many infectious diseases when compared to increases in living standards. A recent book on the Epidemic Intelligence Service of the Centers for Disease Control and Prevention, USA, is used to illustrate the importance of epidemiological surveillance and public health laboratories in past and present efforts to control infectious diseases. On the basis of these publications, the author discusses and proposes solutions for some of the shortcomings the Chilean sanitary services demonstrated when dealing with a recent epidemic of more than 26,000 cases of acute diarrhea in the Northern Chilean city of Antofagasta.

"Los grupos humanos se crean y se constituyen a través de las decisiones que usan para modificar el medio ambiente en que viven".

Rene Dubos.

$\mathrm{E}$ ste comentario utiliza un reciente artículo aparecido en la prensa norteamericana acerca del fracaso de los planes de la Fundación Gates en erradicar la poliomielitis en Asia y África como una base para discutir el, a menudo, limitado rol histórico que han jugado las vacunas en controlar muchas enfermedades infecciosas cuando se les compara con el crecimiento en los estándares de vida. Se emplea un reciente libro acerca del Servicio de Inteligencia de Epidemias del Centers for Disease Control and Prevention, de E.U.A., para ilustrar la importancia de la vigilancia epidemiológica y de los laboratorios públicos de salud en los esfuerzos pasados y actuales para controlar las enfermedades infecciosas. En base a estas publicaciones, el autor discute y propone soluciones para algunos de las deficiencias en los servicios sanitarios chilenos que fueran puestas en evidencia al enfrentar una reciente epidemia de más de 26.000 casos de diarrea aguda en la norteña ciudad chilena de Antofagasta.

Lecturas contemporáneas aparentemente disímiles como la de un artículo en el Wall Street Journal, de la revisión de un libro sobre la historia del programa de inteligencia epidemiológica del Centers for Disease Control and Prevention (CDC) de E.U.A y de las noticias en El Mercurio y otros diarios acerca de la gran epidemia de gastroenteritis en Antofagasta, evocan aspectos diversos y comunes, de la evolución permanente y tan llena de sorpresas de las enfermedades infecciosas.

El artículo del Wall Street Journal: "Gates repiensa su guerra contra la polio" (Wall Street Journal, abril 23, 2010) se refiere a los problemas y los fracasos del programa para la erradicación mundial de la poliomielitis llevado a cabo por la Fundación Gates con el objetivo teórico de hacer desaparecer esta enfermedad de los países del Asia y del África, y del mundo. Según el periodista, el millonario Bill Gates ha llegado a la conclusión, como también han llegado a la misma conclusión personeros de la Organización Mundial de la Salud, respecto de las limitaciones del uso de las vacunas como únicas armas preventivas para éste y otros flagelos. El fracaso del programa de la Fundación Gates para erradicar la poliomielitis en África y Asia ha traído nuevamente al campo de la discusión en salud pública un antiguo debate respecto de lo adecuado y racional del uso de vacunas como el único o el principal método para combatir las enfermedades infecciosas. Además, este debate pone en el tapete de la discusión el concepto de que los fondos que se invierten en campañas vacunales destinadas a la erradicación de una sola enfermedad pudieran ser mejor invertidos en otros aspectos que mejoran también la salud de la población, y que son de más largo alcance y menos publicitarios pero no por eso menos efectivos. Estos aspectos incluyen el mejoramiento de la nutrición y de la vivienda, el aumentar el acceso a la educación y a la salud, el optimizar el saneamiento y la seguridad
Department of Microbiology and Immunology, New York Medical College, Valhalla, NY 10595, EE.UU.

Recibido: 10 de mayo de 2010

Correspondencia a: Felipe Cabello C cabello@nymc.edu 
alimentaria y el crear programas de salud accesibles con la participación democrática de la comunidad a la cual ellos estas dirigidos.

A mi modo de ver, el modelo que persigue al uso de vacunas como la única o la principal herramienta en la lucha contra la enfermedad infecciosa, ha sido siempre un imaginario o pseudo paradigma. Este aparentemente lógico patrón que ha sido usado e impulsado con diversos fines, desde políticos y económicos y hasta propagandísticos, está basado en la ignorancia voluntaria e involuntaria del record histórico que indica que estas enfermedades comenzaron a desaparecer como causa de mortalidad en los países industrializados en una época previa a la introducción de las vacunas y de los antimicrobianos. Esto fue muy bien demostrado por el médico inglés Thomas McKeown en dos de sus obras, "La medicina en la sociedad moderna", (Hafner Publish. Co. NY.), 1966 y "El papel de la medicina. Sueño, ilusión o pesadilla", (Princeton U. Press, NJ), 1979. Obras en las cuales, después de un crítico análisis de las estadísticas de morbilidad y mortalidad en Inglaterra, él concluye que la declinación de la mortalidad por estas enfermedades observada en el siglo XIX y a comienzo del siglo XX se debió al aumento de los niveles de vida (nutrición, educación, vivienda, condiciones de trabajo) y a la introducción de medidas de higiene como la disposición sanitaria de excretas y el control microbiológico del agua y de los alimentos. Factores que él considera de mejoramiento e ingeniería ambiental. Vacunas y antimicrobianos vinieron a ayudar a la disminución de la mortalidad por enfermedades infecciosas cuando la mayor parte de esta declinación ya se había prácticamente producido. Y esto ocurrió así con la tuberculosis, la difteria, la tos convulsiva, las enfermedades entéricas, la neumonía, la viruela, y el sarampión. En mi opinión, el fracaso de los programas de la erradicación de la poliomielitis en Asia y África de la Fundación Gates, a pesar de las ingentes inversiones de dinero descritas en el Wall Street Journal, no hacen sino confirmar una vez más la racionalidad de esta manera de ver estos problemas. Indicando que el aumento de los niveles de vida y el mejoramiento del ambiente, son herramientas muchos más poderosas que las vacunas y los antimicrobianos para disminuir la relevancia de la morbilidad y de la mortalidad por estas enfermedades.

El asentamiento conceptual y definitivo de la teoría microbiana de la enfermedad infecciosa, como resultado del trabajo de Pasteur, Koch y sus colaboradores, hizo posible a fines del siglo XIX el estudio de la epidemiología de las enfermedades trasmisibles con el uso del laboratorio. Esto permitió por primera vez relacionar la existencia de entidades infecciosas presentes en el ambiente y en los vehículos de contagio, con la aparición de microorganismos en el hospedero y el comienzo y desarrollo de patología infecciosa. Pudiendo estos hospederos ser humanos, animales o plantas. El desarrollo del laboratorio de salud publica y de estos conceptos fueron cruciales, para desplegar las medidas de saneamiento ambiental y de la seguridad microbiana del agua de bebida y de los alimentos. La importancia y la revolución que este concepto significó en la epidemiología de la enfermedad infecciosa ha sido recientemente ilustrada por la aparición del libro "Adentro de las epidemias. Los extraordinarios médicos detectives del servicio de inteligencia epidemiológica" del autor Mark Pendergast (Houghton Mifflin Hartcourt NY), 2010. Este libro es una historia de la unidad de inteligencia epidemiológica del CDC de E.U.A. fundada en 1951 y en él se relata de manera dinámica cómo la epidemiología de campo llevada a cabo por esta unidad ha sido la responsable del descubrimiento, de manera efectiva y brillante, de los agentes etiológicos de nuevas enfermedades, incluyendo entre otras la enfermedad de los legionarios, las fiebres producidas por hantavirus, el síndrome de shock tóxico y la enfermedad de Lyme.

En una de las revisiones de este libro se dice algo que, sin lugar a dudas, es muy cierto: que los éxitos de esta unidad de inteligencia epidemiológica ilustran de manera incontrovertible que para los gobiernos es mucho más práctico, desde el punto de vista económico y ético, encontrar la causas y las raíces de las epidemias de enfermedades infecciosas de manera rápida y efectiva antes que estas generen miles de casos cuyo tratamiento signifique gasto de cantidades importantes de dinero y de recursos siempre escasos. Dicho de otra forma, la inversión en salud pública produce grandes ahorros económicos además de ser una forma más humana y ética de aproximarse a estos problemas; con ello se evitan el sufrimiento que la enfermedad y las muertes innecesarias ocasionan.

Es importante señalar que la unidad de inteligencia epidemiológica del CDC se ha beneficiado también de la organizada red de laboratorios microbiológicos locales instalados a nivel de consultorios y hospitales en los barrios, ciudades, condados, estados y regiones de E.U.A. Los laboratorios locales le toman el pulso diariamente a los patógenos aislados de manera rutinaria de pacientes, de animales y de potenciales vehículos de transmisión. Recibiendo la unidad información inmediata acerca de singularidades que pudieran indicar los inicios de un problema, incluyendo desde los comienzos de una epidemia hasta la aparición de resistencia a antimicrobianos en bacterias que hasta ese momento no la expresaban. Esto le ha permitido a la unidad de inteligencia epidemiológica preocuparse también de algunos problemas rutinarios como el de las infecciones intrahospitalarias, la seguridad alimentaria y la contaminación de los medicamentos. Además, la unidad ha podido investigar problemas de salud pública que pueden también estudiarse con la aplicación del método epidemiológico tales como las intoxicaciones, los defectos congénitos, la obesidad y una serie de problemas médicos 
generados en el trabajo. La labor positiva que esta unidad ha llevado a cabo desde su creación en pos de la salud de la población, no sólo de E.U.A. sino que también del mundo, nos ilumina la interpretación de las noticias de El Mercurio y otros diarios que nos informan acerca de la gran epidemia de diarrea aguda que asolara la ciudad de Antofagasta entre marzo y abril de este año.

Analizada la lectura de estas noticias desde un punto de vista técnico, racional y moderno, existen varios aspectos y anomalías que llaman la atención respecto de la evolución de estos procesos biológicos y sociales en Chile, a comienzos del siglo XXI. En primer lugar, es inquietante la ausencia de comunicados, ya sea de la autoridad sanitaria o de la autoridad política, reconociendo la presencia de esta gran epidemia, informando a la población acerca de ella, presentando las medidas efectuadas para interrumpirla y discutiendo las modificaciones sanitarias implementadas para prevenir similares ocurrencias futuras. Mantener a la población informada, además de diagnosticar y de reconocer el problema, no sólo educa sino que evita que versiones alarmistas y frívolas de la prensa distorsionen el origen y el potencial desarrollo de estos hechos. Indudablemente que esta epidemia se ha debido a fallas graves en el control de la calidad de los productos que la industria privada proporciona a la población, incluyendo alimentos y tal vez el agua de bebida. Sin embargo, también esta epidemia es una señal incontrovertible de omisiones peligrosas en la habilidad de los servicios del Estado para controlar rutinariamente la calidad microbiológica del agua de bebida y de los alimentos a los cuales la población accede. Fracasos graves de los organismos del Estado se aprecian también en la manera de enfrentar esta epidemia y de aminorar sus consecuencias. Puesto que ella evolucionó sin pausa, por lo menos por tres o cuatro semanas, elevándose el número de casos de 3.000 a 6.000 , a 12.000 y por último a 26.000 o más. Esto, sin que medida alguna por parte del Estado y de sus servicios interrumpiera su inexorable e inexcusable curso durante este extendido período. El Mercurio y otros medios de comunicación indican que esta epidemia sería producida por norovirus. Sin embargo, esta hipótesis, aunque plausible es difícil de sostener con la información existente, ya que este agente etiológico según los diarios se ha encontrado sólo en 16 casos de los más de 26.000 que esta epidemia presentara. El hecho que este putativo diagnóstico fuera hecho en un número ínfimo de casos por un laboratorio de la Universidad de Chile y no por uno de los laboratorios del Estado, también indicaría las limitaciones de éste para enfrentar estos problemas de manera eficiente, rutinaria y expedita.

Según la prensa, en los últimos días de la evolución de esta epidemia existieron enfrentamientos políticos respecto de la responsabilidad por ella, entre los parlamentarios representando al gobierno saliente y los ejecutivos en sa- lud representando al gobierno entrante. Sin lugar a dudas, el Parlamento es uno de los lugares procedentes donde este tipo de discusión debe llevarse a cabo, como sucede tradicional y cotidianamente en una serie de países en que estos problemas son ventilados con transparencia pública y tratados por el Estado de manera relevante, diestra y eficiente. Es obvio que esta epidemia y las importantes limitaciones del Estado chileno para lidiar con ella y con otros acontecimientos epidemiológicos del pasado en el país debieran ser motivo de preocupación permanente de las organizaciones políticas y del liderazgo político de la nación. Porque ellas atentan contra el bienestar de la mayoría e hipotecan su futuro, ya que la aparición de estos eventos puede ir en aumento como resultado del cambio climático, la globalización y el deterioro ambiental. Es innegable que esta epidemia se ha producido porque existen en el país fallas graves y delicadas en lo que el Dr. McKeown mencionado más arriba llamó los factores ambientales de la enfermedad infecciosa.

Además esta situación se ha agravado por la ausencia de una efectiva organización de inteligencia epidemiológica nacional capaz de reaccionar en forma organizada y resuelta ante la aparición incipiente de estos procesos. Queda claro también de la discusión previa, que en la prevención de estos episodios epidémicos las vacunas como método de salud pública tienen un menguado y reducido rol. Cabría esperar que de la misma manera en que los representantes del Parlamento y del Ejecutivo se han interesado por las causas y la evolución de esta epidemia, se preocuparan de corregir a futuro las serias deficiencias aquí señaladas, responsables en parte de ella. Estas carencias, además de atentar contra la salud de la población son un problema de seguridad nacional, ya que desmejoran notablemente el rol económico de Chile como una probable potencia alimentaria exportadora de alimentos. Generalmente se aduce que Chile es un país que carece de los recursos económicos necesarios para emprender un avance sensato y real en la satisfacción de una plétora de necesidades postergadas de estos aspectos deficitarios de la salud pública. Sin embargo, es relevante comentar aquí que un país que se escuda contra enemigos imaginarios invirtiendo anualmente en armamentos 4.478 millones de dólares, los cuales corresponden al 4\% de su Producto Interno Bruto, bien podría distraer un porcentaje pequeño de esa suma en generar una adecuada estructura con herramientas actualizadas para combatir ahora a un adversario real constituido por la enfermedad infecciosa. Algunos expertos comparan la aparición de epidemias y sus secuelas de efectos negativos sobre la población a catástrofes naturales como los terremotos y temporales. Sugiriendo esta comparación y el debate actual en el país a raíz del reciente terremoto, que existiría una justificación virtuosa para corregir la serie de problemas discutidos en este comentario con fondos obtenidos también de un 
impuesto a las industrias y a la gran minería del cobre. Es indudable además que los miembros y las organizaciones poseedores del conocimiento moderno acerca de estos problemas, como la Sociedad Chilena de Infectología y otras sociedades profesionales afines, debieran tal vez emprender un rol educacional más activo en la sociedad respecto de ellos y de sus probables soluciones. Ya que como lo enunciara tan claramente Rudolf Virchow, uno de los creadores de la patología y de la salud pública modernas hace ya 150 años, "La ciencia enclaustrada es prácticamente inexistente, ya que ella vive sólo para el beneficio estrecho de los individuos que la practican."

Agradecimientos. Agradezco las sugerencias efectuadas por Katia Velázquez M. al texto.

\section{Réplica al Comentario de F. Cabello}

\section{Reply to Comment of F. Cabello}

Recibido: 19 junio 2010

Señor Director:

En su comentario relacionado con el reciente brote masivo de gastroenteritis aguda ocurrido en Antofagasta, el Dr. Felipe Cabello emite su opinión sobre una serie de temas de Salud Pública que sin duda son de alto interés para el país. Toda opinión es válida, y por cierto más aún si es avalada por una trayectoria en la academia como la del Dr. Cabello; lo bello de la academia, sin embargo, es que las opiniones se confrontan y yo me permito disentir de alguna de estas opiniones así como concordar con otras.

En su análisis sobre el rol de las vacunas como herramienta de Salud Pública, me parece que se presenta una visión algo reduccionista y pareciera apuntar (al menos así lo interpreto) a desmerecer el rol de las vacunas como herramienta fundamental de Salud Pública para la prevención de enfermedades infecciosas. Es evidente, que como medida única serían marcadamente insuficientes, y me es difícil pensar en alguien que pudiera proponer una tesis semejante. Me parece por otro lado, que el argumento de hacer competir diferentes medidas de Salud Pública como mejoras en nutrición, vivienda, educación, etc. con inmunizaciones como la plantea el Dr. Cabello, para decidir cuáles medidas se implementan y cuáles no, no debe ser el centro del debate. En la medida que diferentes medidas sean complementarias para el beneficio de una sociedad, todas debieran ser consideradas y evaluadas seriamente. Por cierto, debemos avanzar en una mejor evaluación de costo-efectividad de las diferentes medidas de Salud Pública, incluyendo inmunizaciones para la toma de decisiones. Me permito disentir profundamente con la opinión que parece transmitir el documento, de que las vacunas han tenido poco impacto en la reducción de las enfermedades infecciosas inmunoprevenibles. No creo que alguien pueda negar la evidencia que demuestra el rol de las vacunas en la erradicación de la viruela, eliminación de polio en las Américas, reducción significativa del sarampión, enfermedad invasora por $H$. influenza b, y la rubéola congénita en la mayoría de los países del mundo; la demostrada disminución de la varicela y la enfermedad invasora por S. pneumoniae en países que han incorporado estas vacunas $\mathrm{y}$, más recientemente, el impacto de las vacunas anti-rotavirus en la disminución de enfermedad moderada a grave y muertes asociado a este agente. No son la única razón para este favorable impacto, pero está en los primeros lugares en la lista de medidas de alta efectividad. La dificultad en la erradicación de la polio se debe fundamentalmente a un problema de cobertura vaccinal en países con un sinnúmero de complejidades sociales; no entiendo la racionalidad argumental que propone el Dr. Cabello en esta materia. En el mismo sentido, restarle el mérito que se merecen al desarrollo de la terapia antimicrobiana como estrategia para disminuir la morbi-mortalidad asociada a las enfermedades infecciosas me parece indefendible. Concuerdo con el Dr. Cabello en que mejorar el nivel de vida de la población y del ambiente puede ser de mayor relevancia, pero se olvida quizás el Dr. Cabello que parte de un mejor nivel de vida es el poder contar con más y mejores vacunas y mayor accesibilidad a antimicrobianos efectivos.

En el tema de la vigilancia epidemiológica de enfermedades concuerdo con el Dr. Cabello que el país ha sido poco eficiente en desarrollar una red efectiva de vigilancia para un número importante de enfermedades. Se ha avanzado, y estoy cierto que el Ministerio de Salud-MINSALpodrá dar cuenta de ello. Un ejemplo palpable desde mi ámbito de acción es la implementación de un sistema de vigilancia "en línea" de virus respiratorios basado en centros centinela distribuidos a nivel nacional. Comparto, sin embargo, la percepción de que hay mucho por hacer para modernizar nuestra vigilancia de enfermedades; es evidente que nuestras estadísticas son deficientes y por lo general desfasadas (y por ende poco útiles para favorecer toma de decisiones). El rol del Instituto de Salud Pública 
de Chile, que debiera ser un referente latinoamericano en esta materia y en el estudio microbiológico de brotes entre otras acciones de salud, es desdibujado y requiere urgentemente de un nuevo liderazgo que identifique metas y estrategias que determinen beneficios demostrables para la población. Concuerdo también en que Chile debiera contar con unidades de inteligencia epidemiológica con profesionales capacitados, equipos y recursos capaces de responder rápidamente ante situaciones epidemiológicas determinadas.

El brote masivo sucedido en Antofagasta hace patente una serie de deficiencias de nuestro sistema actual como lo señala el Dr. Cabello. Mi opinión, habiendo participado indirectamente del estudio de este brote por invitación del MINSAL es que la principal falencia es la de un débil liderazgo institucional que dé claridad sobre las responsabilidades, objetivos, métodos, resultados a obtener, uso de la información, y difusión de la información a la comunidad científica y no científica. Falta coordinación entre los diferentes actores a nivel local y central. Por otro lado, falta compromiso de la autoridad en estos temas que se traduce en poca claridad de lo que se persigue y se desea obtener como producto de las vigilancias epidemiológicas a nivel nacional, y en los recursos que se presupuestan para ello. En pocas palabras, se debe trabajar (y para ello se debiera convocar a un número importante de personas del más alto nivel) para redefinir la forma en que como país estructuraremos la Salud Pública del mañana.

Por otro lado, concuerdo en que el Parlamento debiera conocer de estos temas y proponer leyes que modernicen nuestra infraestructura e instituciones. No soy optimista, sin embargo, al menos que se logre un diálogo más fluido y permanente entre personas del quehacer científico-aca- démico con el quehacer político y se busquen estrategias de país para avanzar hacia una verdadera modernización, por sobre las disputas partidistas. Finalmente, puedo decir que la Sociedad Chilena de Infectología, a través de la contribución permanente, desinteresada y ad honorem de sus socios, participa en forma significativa de un número importante de actividades en conjunto con organismos estatales así como en la difusión de temas para la comunidad científica y no científica; siempre es bueno hacer más, pero somos una comunidad relativamente pequeña y la mayoría de sus socios están "sobrevendidos" con el tiempo que ya le dedican a estas actividades; la buena notica es que las nuevas generaciones están demostrando que mantienen este compromiso con la sociedad chilena.

El contar con una Salud Pública moderna que sirva eficientemente al país dependerá, a mi manera de ver, de nuevos liderazgos, mayor compromiso de las autoridades políticas y, concordando con el último párrafo del Dr. Cabello, mediante una estrategia de alianza entre el sistema público y académico -agregaría el sector privado generosa, transparente y con estrictos criterios de calidad y eficiencia.

\author{
Miguel O’Ryan G. \\ Profesor Titular y Director \\ Programa de Microbiología y Micología \\ Instituto de Ciencias Biomédicas \\ Facultad de Medicina \\ Universidad de Chile \\ Actualmente de desempeña como \\ Vicerrector de Investigación y Desarrollo \\ Universidad de Chile
}

